TAIWANESE JOURNAL OF MATHEMATICS

Vol. 3, No. 3, pp. 251-279, September 1999

\title{
SOLVING POLYNOMIAL SYSTEMS BY POLYHEDRAL HOMOTOPIES
}

\author{
Tien-Yien Li*
}

\begin{abstract}
In the last two decades, the homotopy continuation method has been developed into a reliable and efficient numerical algorithm for solving all isolated zeros of polynomial systems. During the last few years, a major computational breakthrough has emerged in the area. Based on the Bernshtein theory on root count, the polyhedral homotopy is established to considerably reduce the number of homotopy paths that need to be traced to find all the isolated roots, making the method much more powerful. This article reports the most recent development of this new method along with future considerations.
\end{abstract}

\section{INTRODUCTION}

Polynomial systems arose quite commonly in many fields of science and engineering, such as formula construction, geometric intersection, inverse kinematics, power flow with PQ-specified bases, computation of equilibrium states, etc.; see [9]. Elimination theory-based methods, most notably the Buchberger algorithm [5] for constructing Gröbner bases, are the classical approach to solving multivariate polynomial systems, but their reliance on symbolic manipulation makes those methods somewhat unsuitable for all but small problems.

In 1977, Garcia and Zangwill [13] and Drexler [10] independently presented theorems suggesting that homotopy continuation could be used to find the full set of isolated zeros of a polynomial system numerically. During the last two decades this method has been developed into a reliable and efficient numerical

Received June 28, 1999.

Communicated by P. Y. Wu.

1991 Mathematics Subject Classification: 65H10, 68Q40, 93B27.

Key words and phrases: Polynomial systems, homotopy continuation, polyhedral homotopy, Bernshtein theory.

${ }^{*}$ Research supported in part by NSF under Grant DMS-9804846. 
algorithm for approximating all isolated zeros of polynomial systems. See [23] for a survey.

Let $P(x)=0$ be a system of $n$ polynomial equations in $n$ unknowns. Denoting $P=\left(p_{1}, \ldots, p_{n}\right)$, we want to find all isolated solutions of

$$
\begin{gathered}
p_{1}\left(x_{1}, \ldots, x_{n}\right)=0 \\
\vdots \\
p_{n}\left(x_{1}, \ldots, x_{n}\right)=0
\end{gathered}
$$

for $x=\left(x_{1}, \ldots, x_{n}\right)$. The classical homotopy continuation method for solving (1) is to define a trivial system $Q(x)=\left(q_{1}(x), \ldots, q_{n}(x)\right)=0$ and then follow the curves in the real variable $t$ which make up the solution set of

$$
0=H(x, t)=(1-t) Q(x)+t P(x) .
$$

More precisely, if the system $Q(x)$, known as the start system, is chosen correctly, the following three properties hold:

- Property 0 (Triviality). The solutions of $Q(x)=0$ are known.

- Property 1 (Smoothness). The solution set of $H(x, t)=0$ for $0 \leq t \leq 1$ consists of a finite number of smooth paths, each parametrized by $t$ in $[0,1)$.

- Property 2 (Accessibility). Every isolated solution of $H(x, 1)=P(x)=0$ can be reached by some path originating at $t=0$. It follows that this path starts at a solution of $H(x, 0)=Q(x)=0$.

When the three properties hold, the solution paths can be followed from the initial points (known because of Property 0 ) at $t=0$ to all solutions of the original problem $P(x)=0$ at $t=1$ using standard numerical techniques [1,2]. A homotopy $H(x, t)=0$ with $H(x, 0)=Q(x)$ and $H(x, 1)=P(x)$, which may not be in the form of (2), is considered to be successful if it satisfies these three properties.

A typical choice $[8,22,24,29,40,41]$ of the system $Q(x)=\left(q_{1}(x), \ldots, q_{n}(x)\right)$ which satisfies Properties 0-2 is,

$$
\begin{gathered}
q_{1}\left(x_{1}, \ldots, x_{n}\right)=a_{1} x_{1}^{d_{1}}-b_{1} \\
\vdots \\
q_{n}\left(x_{1}, \ldots, x_{n}\right)=a_{n} x_{n}^{d_{n}}-b_{n},
\end{gathered}
$$

where $d_{1}, \ldots, d_{n}$ are the degrees of $p_{1}(x), \ldots, p_{n}(x)$ respectively, and $a_{j}, b_{j}$ are random complex numbers (and therefore nonzero with probability one). So in 
one sense, the original problem posed is solved. All solutions of $P(x)=0$ are found at the end of $d_{1} \cdots d_{n}$ paths that make up the solution set of $H(x, t)=$ $0,0 \leq t \leq 1$.

The reason the problem is not satisfactorily solved by the above considerations is the existence of extraneous paths. Although the above method produces $d=d_{1} \cdots d_{n}$ paths since $Q(x)=0$ in (3) has $d$ isolated nonsingular solutions, the system $P(x)=0$ may have fewer than $d$ solutions. We call such a system deficient. In this case, some of the paths produced by the above method will be extraneous paths.

More precisely, even though Properties 0-2 imply that each solution of $P(x)=0$ will lie at the end of a solution path, it is also consistent with these properties that some of the paths may diverge to infinity as the parameter $t$ approaches 1 (the smoothness property rules this out for $t \rightarrow t_{0}<1$ ). In other words, it is quite possible for $Q(x)=0$ to have more solutions than $P(x)=0$. In this case, some of the paths leading from roots of $Q(x)=0$ are extraneous, and diverge to infinity when $t \rightarrow 1$ (see Figure 1 ).

Empirically, we find that most systems arising in applications are deficient. A great majority of the systems have fewer than, and in some cases only a small fraction of, the expected number of solutions. For a typical example of this sort, let us look at the following Cassou-Nogues system

FIG. 1. 


$$
\begin{aligned}
p_{1}= & 15 b^{4} c d^{2}+6 b^{4} c^{3}+21 b^{4} c^{2} d-144 b^{2} c-8 b^{2} c^{2} e \\
& -28 b^{2} c d e-648 b^{2} d+36 b^{2} d^{2} e+9 b^{4} d^{3}-120, \\
p_{2}= & 30 b^{4} c^{3} d-32 c d e^{2}-720 b^{2} c d-24 b^{2} c^{3} e-432 b^{2} c^{2}+576 c e-576 d e \\
& +16 b^{2} c d^{2} e+16 d^{2} e^{2}+16 c^{2} e^{2}+9 b^{4} c^{4}+39 b^{4} c^{2} d^{2}+18 b^{4} c d^{3} \\
& -432 b^{2} d^{2}+24 b^{2} d^{3} e-16 b^{2} c^{2} d e-240 c+5184, \\
p_{3}= & 216 b^{2} c d-162 b^{2} d^{2}-81 b^{2} c^{2}+1008 c e-1008 d e+15 b^{2} c^{2} d e \\
& -15 b^{2} c^{3} e-80 c d e^{2}+40 d^{2} e^{2}+40 c^{2} e^{2}+5184, \\
p_{4}= & 4 b^{2} c d-3 b^{2} d^{2}-4 b^{2} c^{2}+22 c e-22 d e+261 .
\end{aligned}
$$

Since $d_{1}=7, d_{2}=8, d_{3}=6$ and $d_{4}=4$ for this system, the system $Q(x)$ in (3) will produce $d_{1} \times d_{2} \times d_{3} \times d_{4}=7 \times 8 \times 6 \times 4=1344$ paths for the homotopy in (2). However, the system (4) has only 16 isolated zeros. Consequently, a major fraction of the paths are extraneous. Sending out 1344 paths in search of 16 solutions is a highly wasteful computation.

The choice of $Q(x)$ in (3) to solve the system $P(x)=0$ requires an amount of computational effort proportional to $d_{1} \cdots d_{n}$, known as the Bézout number, which bounds the number of isolated zeros, counting multiplicities, of $P(x)$ in $C^{n}[36]$. We wish to derive methods for solving deficient systems for which the computational effort is instead proportional to the actual number of solutions.

In the last few years, a major computational breakthrough has emerged in the area. The new idea takes a great advantage of the Bernshtein theory [4] which provides a much tighter bound, compared to the Bézout bound, for the number of isolated zeros of $P(x)$ in the algebraic tori $\left(C^{*}\right)^{n}$, where $C^{*}=C \backslash\{0\}$. The so-called polyhedral homotopy [17] is then established for the new method and the homotopy paths so produced are much fewer. Accordingly, the required computation effort is considerably reduced. The new algorithm is very promising. In particular, for polynomial systems without special structures, the new algorithm outperformed the existing methods by a big margin.

In this article, we report some new developments of this method along with the necessary tools. Solving polynomial systems by the polyhedral homotopy in the new method consists of two major steps:

(A) Solve a new polynomial system with the same monomials as the originally given polynomial system, but with randomly chosen coefficients.

(B) The new polynomial system is then used as the start system in the linear homotopy in (2) to solve the original system.

We divide our discussion on solving polynomial systems with randomly chosen coefficients at Step (A) in Sections 2, 3, and 4. In Section 5, the 
cheater's homotopy that uses the polynomial system solved in Step (A) as the start system in the linear homotopy in (2) is described for Step (B). Further considerations of the method as well as a few remarks will be presented in Section 6 .

\section{The Bernshtein Theory}

The Bernshtein theory on root count of polynomial systems is essential for our attempt to reduce the number of homotopy curves need to be traced when the homotopy continuation method is employed to find all the isolated zeros of polynomial systems.

Let the given polynomial system be $P(x)=\left(p_{1}(x), \ldots, p_{n}(x)\right) \in C[x]$, where $x=\left(x_{1}, \ldots, x_{n}\right)$. With $x^{a}=x_{1}^{a_{1}} \ldots x_{n}^{a_{n}}$ where $a=\left(a_{1}, \ldots, a_{n}\right)$, write

$$
\begin{aligned}
& p_{1}(x)=\sum_{a \in S_{1}} c_{1, a}^{*} x^{a}, \\
& \vdots \\
& p_{n}(x)=\sum_{a \in S_{n}} c_{n, a}^{*} x^{a},
\end{aligned}
$$

where $S_{1}, \ldots, S_{n}$ are fixed subsets of $N^{n}$ with cardinalities $k_{j}=\# S_{j}$, and $c_{j, a}^{*} \in C^{*}(=C \backslash\{0\})$ for $a \in S_{j}, j=1, \ldots, n$. We call $S_{j}$ the support of $p_{j}(x)$, denoted by $\operatorname{supp}\left(p_{j}\right)$, its convex hull $Q_{j}=\operatorname{conv}\left(S_{j}\right)$ in $R^{n}$ the Newton polytope of $p_{j}$, and $S=\left(S_{1}, \ldots, S_{n}\right)$ the support of $P(x)$, denoted by $\operatorname{supp}(P)$. where

We now embed the system (5) in the systems $P(c, x)=\left(p_{1}(c, x), \ldots, p_{n}(c, x)\right)$,

$$
\begin{gathered}
p_{1}(c, x)=\sum_{a \in S_{1}} c_{1, a} x^{a}, \\
\vdots \\
p_{n}(c, x)=\sum_{a \in S_{n}} c_{n, a} x^{a},
\end{gathered}
$$

and the coefficients $c_{j, a}$ with $a \in S_{j}$, for $j=1, \ldots, n$ are taken to be a set of $M \equiv k_{1}+\cdots+k_{n}$ variables. Namely, the system $P(x)$ in (5) is considered as a system in (6) corresponding to a set of specified values of coefficients $\bar{c}=\left(c_{j}^{*}, a\right)$ or $P(x)=P(\bar{c}, x)$.

In the rest of the paper, the total number of isolated zeros, counting multiplicities, of a polynomial system will be referred to as the root count of the system.

Lemma 2.1. [16] For polynomial systems $P(c, x)$ in (6), there exists a polynomial system $G(c)=\left(g_{1}(c), \ldots, g_{n}(c)\right)$ in the variables $c=\left(c_{j}, a\right)$ for 
$a \in S_{j}$ and $j=1, \ldots, n$ such that for those coefficients $\bar{c}=\left(c_{j}^{*}, a\right)$ for which $G(\bar{c}) \neq 0$, the root count in $\left(C^{*}\right)^{n}$ of the corresponding polynomial systems in (6) is a fixed number, and the root count in $\left(C^{*}\right)^{n}$ of any other polynomial systems in (6) is bounded above by this number.

Remark 2.2. Since the zeros of the polynomial system $G(c)$ in the above lemma form an algebraic set with dimension smaller than $M$, its complement is open and dense with full measure in $C^{M}$. Therefore, with probability one, $G(\bar{c}) \neq 0$ for randomly chosen coefficients $\bar{c}=\left(c_{j}^{*}, a\right) \in C^{M}$. Hence, polynomial systems $P(\bar{c}, x)$ in (6) with $G(\bar{c}) \neq 0$ are said to be in general position.

A simple example that illustrates the assertion of Lemma 2.1 above is the following $2 \times 2$ linear systems:

$$
\begin{aligned}
& c_{11} x_{1}+c_{12} x_{2}=b_{1}, \\
& c_{21} x_{1}+c_{22} x_{2}=b_{2} .
\end{aligned}
$$

Here, $c=\left(c_{11}, c_{12}, c_{21}, c_{22},-b_{1},-b_{2}\right)$. Let

$$
G(c)=\operatorname{det}\left(\begin{array}{ll}
c_{11} & c_{12} \\
c_{21} & c_{22}
\end{array}\right) \times \operatorname{det}\left(\begin{array}{ll}
c_{11} & b_{1} \\
c_{21} & b_{2}
\end{array}\right) \times \operatorname{det}\left(\begin{array}{ll}
b_{1} & c_{12} \\
b_{2} & c_{22}
\end{array}\right) .
$$

Then, when the coefficient $\bar{c}=\left(c_{11}^{*}, c_{12}^{*}, c_{21}^{*}, c_{22}^{*},-b_{1}^{*},-b_{2}^{*}\right)$ satisfies $G(\bar{c})=0$, its corresponding system in $(7)$ has no isolated solution in $\left(C^{*}\right)^{2}$; otherwise, the system has a unique solution in $\left(C^{*}\right)^{2}$.

Theorem 2.3. ([4], Theorem A) The root count in $\left(C^{*}\right)^{n}$ for a polynomial system $P(x)=\left(p_{1}(x), \ldots, p_{n}(x)\right)$ in general position equals to the mixed volume of its support.

The terminology used in this theorem needs explanation. For nonnegative variables $\lambda_{1}, \ldots, \lambda_{n}$ and the Newton polytopes $Q_{j}$ of $p_{j}$ for $j=1, \ldots, n$, let $\lambda_{1} Q_{1}+\ldots+\lambda_{n} Q_{n}$ denote the Minkowski sum of $\lambda_{1} Q_{1}, \ldots, \lambda_{n} Q_{n}$, that is,

$$
\lambda_{1} Q_{1}+\ldots+\lambda_{n} Q_{n}=\left\{\lambda_{1} r_{1}+\ldots+\lambda_{n} r_{n} \mid r_{j} \in Q_{j}, j=1, \ldots, n\right\} .
$$

It can be shown that the $n$-dimensional volume of this polytope $\operatorname{Vol}_{n}\left(\lambda_{1} Q_{1}+\right.$ $\left.\ldots+\lambda_{n} Q_{n}\right)$ is a homogeneous polynomial of degree $n$ in $\lambda_{1}, \ldots, \lambda_{n}$. The coefficient of the term $\lambda_{1} \times \ldots \times \lambda_{n}$ in this homogeneous polynomial is called the mixed volume of the polytopes $Q_{1}, \ldots, Q_{n}$, denoted by $\mathcal{M}\left(Q_{1}, \ldots, Q_{n}\right)$, or the mixed volume of the support of the system $P(x)=\left(p_{1}(x), \ldots, p_{n}(x)\right)$, denoted by $\mathcal{M}\left(S_{1}, \ldots, S_{n}\right)$ where $S_{j}=\operatorname{supp}\left(p_{j}\right)$ for $j=1, \ldots, n$. Sometimes, when no ambiguities exist, it is called the mixed volume of $P(x)$. 
In [6], this root count was nicknamed the BKK bound after its inventors, Bernshtein [4], Kushnirenko [21] and Khovanskii [20]. In general, it provides a much tighter bound compared to variant Bézout bounds $[31,36]$. An apparent limitation of the theorem is that it only counts the isolated zeros of polynomial systems in $\left(C^{*}\right)^{n}$ rather than all the isolated zeros in the affine space $C^{n}$. For the purpose of finding all the isolated zeros of a polynomial system in $C^{n}$, a generalized version of the assertion in the theorem which counts the roots in $C^{n}$ is strongly desirable. This problem was first attempted in [34] by introducing the notion of the shadowed sets and a bound for the root count in $C^{n}$ was obtained. Later, a significantly much tighter bound was discovered in the following theorem.

Theorem 2.4. [28] The root count in $C^{n}$ of a polynomial system $P(x)=$ $\left(p_{1}(x), \ldots, p_{n}(x)\right)$ with supports $S_{j}=\operatorname{supp}\left(p_{j}\right), j=1, \ldots, n$, is bounded above by the mixed volume $\mathcal{M}\left(S_{1} \cup\{0\}, \ldots, S_{n} \bigcup\{0\}\right)$.

In other words, the theorem says that the root count in $C^{n}$ of a polynomial system $P(x)=\left(p_{1}(x), \ldots, p_{n}(x)\right)$ is bounded above by the root count in $\left(C^{*}\right)^{n}$ of the polynomial system $\bar{P}(x)$ in general position obtained by augmenting constant terms to those $p_{j}^{\prime} s$ in $P(x)$ which do not have them. As a corollary, when $0 \in S_{j}$ for all $j=1, \ldots, n$, namely, all $p_{j}(x)$ in $P(x)$ have constant terms, then the mixed volume of $P(x)$ also serves as a bound for the root count of $P(x)$ in $C^{n}$, more than just a bound for the root count in $\left(C^{*}\right)^{n}$ as Theorem 2.3 asserts.

This theorem was further extended in several different ways $[18,35]$.

\section{The Polyhedral Homotopy}

In light of Theorem 2.4 given in the last section, to find all isolated zeros of a given polynomial system $P(x)=\left(p_{1}(x), \ldots, p_{n}(x)\right)$ in $C^{n}$ with support $S=$ $\left(S_{1}, \ldots, S_{n}\right)$, we first augment the monomial $x^{0}(=1)$ to those $p_{i}$ 's which do not have constant terms. Followed by choosing coefficients of all the monomials at random, a new system $Q(x)=\left(q_{1}(x), \ldots, q_{n}(x)\right)$ with support $S^{\prime}=\left(S_{1}^{\prime}, \ldots, S_{n}^{\prime}\right)$ is obtained, where, of course, $S_{j}^{\prime}=S_{j} \cup\{0\}$ for $j=1, \ldots, n$. We want to solve this system in the first place, and the details will be discussed in this section. Afterwards, in Section 5, this system will be used as the start system to solve $P(x)=0$ via linear homotopy in (2).

To begin, we write 


$$
Q(x)=\left\{\begin{array}{ccc}
q_{1}(x) & =\sum_{a \in S_{1}^{\prime}} \bar{c}_{1, a} x^{a}, \\
\vdots & \\
q_{n}(x)= & \sum_{a \in S_{n}^{\prime}} \bar{c}_{n, a} x^{a} .
\end{array}\right.
$$

Since all those coefficients $\bar{c}_{j, a}$ for $a \in S_{j}^{\prime}$ and $j=1, \ldots, n$ are chosen at random, this system may be considered as a system in general position. Namely, there exists a polynomial system

$$
G(c)=\left(g_{1}(c), \ldots, g_{n}(c)\right)
$$

in the variables $c=\left(c_{j, a}\right)$, for $a \in S_{j}^{\prime}$ and $j=1, \ldots, n$, such that $G(\bar{c}) \neq 0$ for the set of coefficients $\bar{c}=\left(\bar{c}_{j, a}\right)$ in (8), and such polynomial systems reach the maximum root count in $\left(C^{*}\right)^{n}$ for the support $S^{\prime}=\left(S_{1}^{\prime}, \ldots, S_{n}^{\prime}\right)$.

Let $t$ denote a new complex variable and consider the polynomial system $\hat{Q}(x, t)=\left(\hat{q}_{1}(x, t), \ldots, \hat{q}_{n}(x, t)\right)$ in the $n+1$ variables $(x, t)$ given by

$$
\hat{Q}(x, t)=\left\{\begin{array}{ccc}
\hat{q}_{1}(x, t) & =\sum_{a \in S_{1}^{\prime}} \bar{c}_{1, a} x^{a} t^{w_{1}(a)}, \\
\vdots & \\
\hat{q}_{n}(x, t)= & \sum_{a \in S_{n}^{\prime}} \bar{c}_{n, a} x^{a} t^{w_{n}(a)},
\end{array}\right.
$$

where each $w_{j}: S_{j}^{\prime} \rightarrow R$ for $j=1, \ldots, n$ is chosen generically. For a fixed $t_{0}$, we rewrite the system in (10) as

$$
\hat{Q}\left(x, t_{0}\right)=\left\{\begin{array}{c}
\hat{q}_{1}\left(x, t_{0}\right)=\sum_{a \in S_{1}^{\prime}}\left(\bar{c}_{1, a} t_{0}^{w_{1}(a)}\right) x^{a}, \\
\vdots \\
\hat{q}_{n}\left(x, t_{0}\right)=\sum_{a \in S_{n}^{\prime}}\left(\bar{c}_{n, a} t_{0}^{w_{n}(a)}\right) x^{a} .
\end{array}\right.
$$

This system is in general position if for $G(c)$ in (9),

$$
T\left(t_{0}\right) \equiv G\left(\bar{c}_{j, a} t_{0}^{w_{j}(a)}\right) \neq 0, \text { for } a \in S_{j}^{\prime} \text { and } j=1, \ldots, n .
$$

The equation $T(t)=0$ can have at most finitely many solutions, since $T(t)$ is not identically 0 because $T(1)=G\left(\bar{c}_{j, a}\right) \neq 0$. Let

$$
t_{1}=r_{1} e^{i \theta_{1}}, \ldots, t_{k}=r_{k} e^{i \theta_{k}}
$$


be the solutions of $T(t)=0$. Then, for any $\theta \neq \theta_{j}$ for $j=1, \ldots, k$, the systems $\bar{Q}(x, t)=\left(\bar{q}_{1}(x, t), \ldots, \bar{q}_{n}(x, t)\right)$ given by

$$
\bar{Q}(x, t)=\left\{\begin{array}{cc}
\bar{q}_{1}(x, t)= & \sum_{a \in S_{1}^{\prime}}\left(\bar{c}_{1, a} e^{i w_{1}(a) \theta}\right) x^{a} t^{w_{1}(a)}, \\
\vdots & \\
\bar{q}_{n}(x, t)=\sum_{a \in S_{n}^{\prime}}\left(\bar{c}_{n, a} e^{i w_{n}(a) \theta}\right) x^{a} t^{w_{n}(a)},
\end{array}\right.
$$

are in general position for all $t>0$ because

$$
\bar{c}_{j, a} e^{i w_{j}(a) \theta} t^{w_{j}(a)}=\bar{c}_{j, a}\left(t e^{i \theta}\right)^{w_{j}(a)}
$$

and

$$
G\left(\bar{c}_{j, a}\left(t e^{i \theta}\right)^{w_{j}(a)}\right)=T\left(t e^{i \theta}\right) \neq 0 .
$$

Therefore, without loss of generality, (choose an angle $\theta$ at random and change the coefficients $\bar{c}_{j, a}$ to $\bar{c}_{j, a} e^{i w_{j}(a) \theta}$ if necessary) we may suppose the systems $\hat{Q}(x, t)$ in (10) are in general position for all $t>0$. Together with Lemma 2.1 given in the last section, it follows that for all $t>0$ the systems $\hat{Q}(x, t)$ in (10) have the same number of isolated zeros in $\left(C^{*}\right)^{n}$. This number, say $k$, should equal to the mixed volume of the support of $Q(x)$ in (8) by Theorem 2.3. We shall skip this fact temporarily and will reach this assertion at the end of this section.

Now, consider $\hat{Q}(x, t)=0$ as a homotopy defined on $\left(C^{*}\right)^{n} \times[0,1]$, known as the polyhedral homotopy. We have $\hat{Q}(x, 1)=Q(x)$, and the zero set of this homotopy is made up of $k$ homotopy paths, say, $x^{(1)}(t), \ldots, x^{(k)}(t)$, since for each $t, 0<t \leq 1, \hat{Q}(x, t)$ has exactly $k$ isolated zeros from the argument given above. Since each $\hat{q}_{j}(x, t)$ has nonzero constant term for all $j=1, \ldots, n$, by a standard application of generalized Sard's Theorem [7], all those homotopy paths are smooth with no bifurcations. Therefore, both Property 1 (Smoothness) and Property 2 (Accessibility) introduced in Section 1 hold for this homotopy. However, at $t=0, \hat{Q}(x, 0) \equiv 0$; see Figure 2. Consequently, the starting points $x^{(1)}(0), \ldots, x^{(k)}(0)$ of those homotopy paths cannot be identified, causing the breakdown of the standard homotopy continuation algorithm. This major obstacle can be overcome by the device we describe below.

For $\alpha=\left(\alpha_{1}, \ldots, \alpha_{n}\right) \in R^{n}$, consider the transformation $y=t^{-\alpha} x$ defined by

$$
\begin{aligned}
y_{1} & =t^{-\alpha_{1}} x_{1}, \\
& \vdots \\
y_{n} & =t^{-\alpha_{n}} x_{n} .
\end{aligned}
$$


FIG. 2.

For $a=\left(a_{1}, \ldots, a_{n}\right) \in N^{n}$, we have

$$
\begin{aligned}
x^{a} & =x_{1}^{a_{1}} \cdots x_{n}^{a_{n}} \\
& =\left(y_{1} t^{\alpha_{1}}\right)^{a_{1}} \cdots\left(y_{n} t^{\alpha_{n}}\right)^{a_{n}} \\
& =y_{1}^{a_{1}} \cdots y_{n}^{a_{n}} t^{\alpha_{1} a_{n}+\cdots+\alpha_{n} a_{n}} \\
& =y^{a} t^{\langle\alpha, a\rangle} .
\end{aligned}
$$

Here, $\langle\cdot, \cdot\rangle$ stands for the usual inner product in $R^{n}$. Substituting (12) into (10) yields, for $j=1, \ldots, n$,

$$
\begin{aligned}
h_{j}(y, t) & \equiv \hat{q}_{j}\left(y t^{\alpha}, t\right)=\sum_{a \in S_{j}^{\prime}} \bar{c}_{j, a} y^{a} t^{\langle\alpha, a\rangle} t^{w_{j}(a)} \\
& =\sum_{a \in S_{j}^{\prime}} \bar{c}_{j, a} y^{a} t^{\left\langle(\alpha, 1),\left(a, w_{j}(a)\right)\right\rangle} \\
& =\sum_{a \in S_{j}^{\prime}} \bar{c}_{j, a} y^{a} t^{\langle\hat{\alpha}, \hat{a}\rangle},
\end{aligned}
$$

where $\hat{\alpha}=(\alpha, 1) \in R^{n+1}$, and $\hat{a}=\left(a, w_{j}(a)\right)$ for $a \in S_{j}^{\prime}$. The new homotopy

$$
H(y, t)=\left(h\left(y_{1}, t\right), \ldots, h_{n}(y, t)\right)=0
$$

retains most of the properties of the homotopy $\hat{Q}(x, t)=0$, in particular, $H(y, 1)=\hat{Q}(y, 1)=Q(y)$ and both Properties 1 (Smoothness) and 2 (Accessibility) stand. Let 


$$
\beta_{j}=\min _{a \in S_{j}^{\prime}}\langle\hat{\alpha}, \hat{a}\rangle, \quad j=1, \ldots, n,
$$

and define the homotopy

$$
H_{\alpha}(y, t)=\left(h_{1}^{\alpha}(y, t), \ldots, h_{n}^{\alpha}(y, t)\right)=0
$$

on $\left(C^{*}\right)^{n} \times[0,1]$, where, for $j=1, \ldots, n$,

$$
\begin{aligned}
h_{j}^{\alpha}(y, t) & \equiv t^{-\beta_{j}} h_{j}(y, t)=\sum_{\substack{a \in S_{j}^{\prime} \\
j \in S_{j}^{\prime} \\
\langle\hat{\alpha}, \hat{a}\rangle=\beta_{j}}} \bar{c}_{j, a} y^{a} t^{\langle\hat{\alpha}, \hat{a}\rangle-\beta_{j}} y^{a}+\sum_{\substack{a \in S_{j}^{\prime} \\
\langle\hat{\alpha}, \hat{a}\rangle>\beta_{j}}} \bar{c}_{j, a} y^{a} t^{\langle\hat{\alpha}, \hat{a}\rangle-\beta_{j}} .
\end{aligned}
$$

Evidently, for any path $\tilde{y}(t)$ defined on $[0,1]$, we have, for all $t>0$,

$$
H_{\alpha}(\tilde{y}(t), t)=0 \quad \Longleftrightarrow \quad H(\tilde{y}(t), t)=0 .
$$

Therefore, the zero set of $H_{\alpha}(y, t)=0$ consists of the same homotopy paths of the homotopy $H(y, t)=0$ in (14). The difference is, the starting points of the homotopy paths considered in the homotopy $H_{\alpha}(y, t)=0$ are solutions of the system

$$
H_{\alpha}(y, 0)=\left\{\begin{array}{c}
h_{1}^{\alpha}(y, 0)=\sum_{\substack{a \in S_{1}^{\prime} \\
\langle\hat{\alpha}, \hat{a}\rangle=\beta_{1}}} \bar{c}_{1, a} y^{a}=0, \\
\vdots \\
h_{n}^{\alpha}(y, 0)=\sum_{\substack{a \in S_{n}^{\prime} \\
\langle\hat{\alpha}, \hat{a}\rangle=\beta_{n}}} \bar{c}_{n, a} y^{a}=0 .
\end{array}\right.
$$

As indicated below, when this system is in certain desired form, its isolated nonsingular solutions that lie in $\left(C^{*}\right)^{n}$ can be constructively identified. In those situations, Property 0 (Triviality) becomes partially valid for the homotopy paths that emanate from those nonsingular solutions of (18) in $\left(C^{*}\right)^{n}$, and we may follow those paths to reach a partial set of isolated zeros of $Q(y)$ at $t=1$.

The system (18) is known as the binomial system if each $h_{j}^{\alpha}(y, 0)$ consists of exactly two terms, that is,

$$
\begin{gathered}
h_{1}^{\alpha}(y, 0)=c_{11} y^{a_{1}^{(1)}}+c_{12} y^{a_{2}^{(1)}}=0, \\
\vdots \\
h_{n}^{\alpha}(y, 0)=c_{n 1} y^{a_{1}^{(n)}}+c_{n 2} y^{a_{2}^{(n)}}=0,
\end{gathered}
$$


where $a_{l}^{(j)} \in S_{j}^{\prime}$ and $c_{j l}=\bar{c}_{j, a_{l}}$ for $j=1, \ldots, n$ and $l=1,2$.

Proposition 3.1. The binomial system in (19) has

$$
k_{\alpha} \equiv\left|\operatorname{det}\left(\begin{array}{c}
a_{1}^{(1)}-a_{2}^{(1)} \\
\vdots \\
a_{1}^{(n)}-a_{2}^{(n)}
\end{array}\right)\right|
$$

nonsingular solutions in $\left(C^{*}\right)^{n}$.

The proof of this proposition is constructive and therefore provides an algorithm for solving the binomial system $(18)$ in $\left(C^{*}\right)^{n}$. We will come back to this matter in the next section.

In summary, for given $\alpha=\left(\alpha_{1}, \ldots, \alpha_{n}\right) \in R^{n}$, by changing variables $y=$ $t^{-\alpha} x$, as in (11), in the homotopy $\hat{Q}(x, t)=\left(\hat{q}_{1}(x, t), \ldots, \hat{q}_{n}(x, t)\right)=0$ in $(10)$, the homotopy $H(y, t)=\left(h_{1}(y, t), \ldots, h_{n}(y, t)\right)=0$ in (14) is obtained, where $h_{j}(y, t)=\hat{q}_{j}\left(y t^{\alpha}, t\right)$. Followed by factoring out the lowest power $t^{\beta_{j}}$ of $t$ among all monomials in each individual $h_{j}(y, t)=0$ for $j=1, \ldots, n$, we arrive at the homotopy $H_{\alpha}(y, t)=0$ in (16). When the start system $H_{\alpha}(y, 0)=0$ of this homotopy is binomial, its nonsingular solutions in $\left(C^{*}\right)^{n}, k_{\alpha}$ (as given in (20)) of them, become available. We may then follow those homotopy paths of $H_{\alpha}(y, t)=0$ originated from those $k_{\alpha}$ regular solutions of $H_{\alpha}(y, 0)=0$ in $\left(C^{*}\right)^{n}$, and reach $k_{\alpha}$ isolated zeros of $Q(y)$ at $t=1$. Worth noting here is the fact that the system $Q(x)$, or $Q(y)$, stays invariant at $t=1$ during the process. See Figure 3.

Now, the existence of $\alpha \in R^{n}$ for which the start system $H_{\alpha}(y, 0)=0$ is binomial is warranted by the following

Proposition 3.2. For all the real functions $w_{j}: S_{j}^{\prime} \rightarrow R, j=1, \ldots, n$, being generically chosen, there must exist $\alpha \in R^{n}$ for which the start system $H_{\alpha}(y, 0)=0$ of the homotopy $H_{\alpha}(y, t)=0$ in (16) is binomial with a nonempty set of nonsingular solutions in $\left(C^{*}\right)^{n}$, i.e., $k_{\alpha} \neq 0$ in (20).

The assertion of this proposition was proved implicitly in [17] with terminologies and machineries developed in combinatorial geometry, such as, random liftings, fine mixed subdivisions, lower facets of convex polytopes, etc.; see also [23]. Here, we elect to reinterpret the result without those specialized terms.

Now, different $\alpha \in R^{n}$ given in Proposition 3.2 leads to different homotopy $H_{\alpha}(y, t)=0$ in (16). Henceforth, following homotopy paths of those different homotopies will reach different sets of isolated zeros of $Q(y)$. By taking the 
Puiseux series expansions of those homotopy paths of $H_{\alpha}(y, t)=0$ originated at $\left(C^{*}\right)^{n}$ into consideration, it is not hard to see that those different sets of

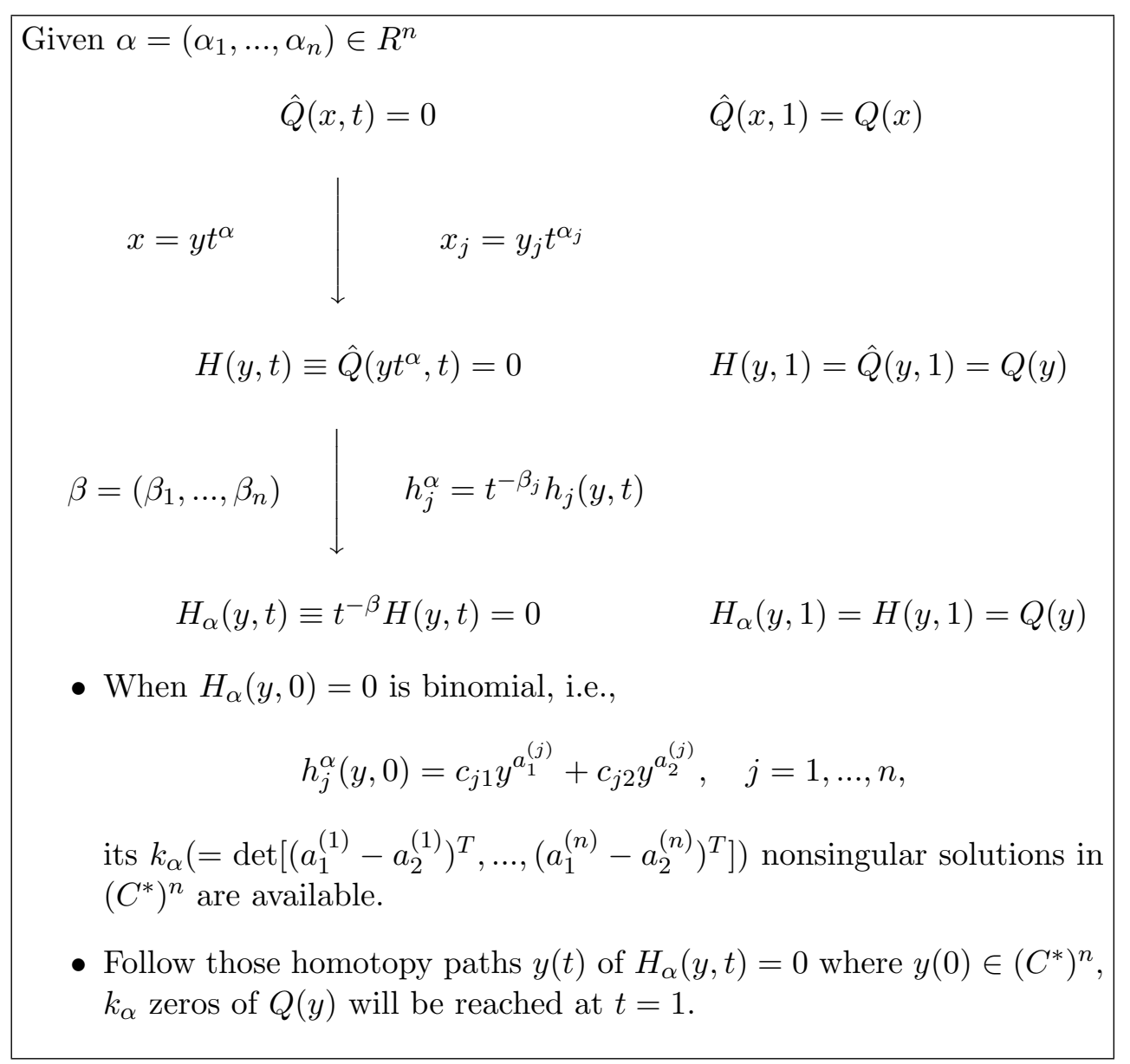

FIG. 3

isolated zeros of $Q(y)$ reached by different sets of homotopy paths are actually disjoint from each other. Most importantly, it can be shown that every isolated zero of $Q(y)$ can be obtained this way by following certain homotopy curve of the homotopy $H_{\alpha}(y, t)=0$ associated with certain $\alpha \in R^{n}$ given by Proposition 3.2. Thus the total number of isolated zeros of $Q(y)$ must equal to the sum of those $k_{\alpha}$ 's corresponding to all the possible $\alpha$ 's provided by Proposition 3.2 , respectively. In [17], it was shown that this sum actually equal to the mixed volume of $Q(y)$. This yields an alternative proof of Theorem 3.2, which is very different from Bernshtein's original approach [4]. 


\section{The Algorithms}

Summarizing the details of the polyhedral homotopy method described in the last section for solving all the isolated zeros of the system $Q(x)=$ $\left(q_{1}(x), \ldots, q_{n}(x)\right)$ in $(8)$, where

$$
\begin{aligned}
q_{1}(x) & =\sum_{a \in S_{1}^{\prime}} \bar{c}_{1, a} x^{a}, \\
\vdots & \\
q_{n}(x) & =\sum_{a \in S_{n}^{\prime}} \bar{c}_{n, a} x^{a},
\end{aligned}
$$

we have the following procedures:

Step 0. Choose a set of real valued functions, $w_{j}: S_{j}^{\prime} \rightarrow R, j=1, \ldots, n$, generically. In fact, since all $S_{j}^{\prime}$ are finite, only finitely many real random numbers need to be identified.

Step 1. Find all the possible $\alpha \in R^{n}$ given in Proposition 3.2, and for each $\alpha$ find a set of pairs of distinct vectors

$$
A_{\alpha}=\left\{\left(a_{1}^{(j)}, a_{2}^{(j)}\right) \mid a_{l}^{(j)} \in S_{j}^{\prime}, \text { for } j=1, \ldots, n \text { and } l=1,2\right\}
$$

such that

$$
k_{\alpha} \equiv\left|\operatorname{det}\left(\begin{array}{c}
a_{1}^{(1)}-a_{2}^{(1)} \\
\vdots \\
a_{1}^{(n)}-a_{2}^{(n)}
\end{array}\right)\right| \neq 0 .
$$

More precisely, we look for $\alpha \in R^{n}$ and its associated set of vectors $A_{\alpha}$ in (21) for which the start system $H_{\alpha}(y, 0)=0$ of the homotopy $H_{\alpha}(y, t)=\left(h_{1}^{\alpha}(y, t), \ldots, h_{n}^{\alpha}(y, t)\right)=0$, where for $j=1, \ldots, n$,

$$
h_{j}^{\alpha}(y, t)=\sum_{a \in S_{j}^{\prime}} \bar{c}_{j, a} y^{a} t^{\langle\hat{\alpha}, \hat{a}\rangle-\beta_{j}}
$$

with $\langle\hat{\alpha}, \hat{a}\rangle=\left\langle(\alpha, 1),\left(a, w_{j}(a)\right)\right\rangle=\langle\alpha, a\rangle+w_{j}(a)$ and $\beta_{j}=\min _{a \in S_{j}^{\prime}}\langle\hat{\alpha}, \hat{a}\rangle$, is in the following binomial form:

$$
\begin{gathered}
h_{1}^{\alpha}(y, 0)=c_{11} y^{a_{1}^{(1)}}+c_{12} y^{a_{2}^{(1)}}=0, \\
\vdots \\
h_{n}^{\alpha}(y, 0)=c_{n 1} y^{a_{1}^{(n)}}+c_{n 2} y^{a_{2}^{(n)}}=0,
\end{gathered}
$$

where $c_{j l}=\bar{c}_{j, a_{l}^{(j)}}$ for $j=1, \ldots, n$ and $l=1,2$. 
Step 2. For each $\alpha$ obtained in Step 1, identify the $k_{\alpha}$, as in (22), nonsingular solutions of the binomial system $(24)$ in $\left(C^{*}\right)^{n}$.

Step 3. For each $\alpha$, follow $k_{\alpha}$ homotopy paths of the homotopy $H_{\alpha}(y, t)=0$ in (23) with starting points in $\left(C^{*}\right)^{n}$ obtained in Step 2. At $t=1$, they reach $k_{\alpha}$ isolated zeros of $Q(y)$, and when $\alpha$ is exhausted we find all the isolated zeros of $Q(y)$.

Among these steps, the algorithms for Steps 0 and 3 are standard application of existing methods. Hence, we only discuss the details of the algorithms for Steps 1 and 2 in the rest of this section,

First of all, $\alpha \in R^{n}$ in Step 1 must satisfy the following conditions:

(i) $\left\langle\hat{\alpha}, \hat{a}_{1}^{(j)}\right\rangle=\left\langle\hat{\alpha}, \hat{a}_{2}^{(j)}\right\rangle=\beta_{j}$,

(ii) $\beta_{j}<\langle\hat{\alpha}, \hat{a}\rangle$ for all $a \in S_{j}^{\prime}, \quad j=1, \ldots, n$,

and the matrix

$$
\left(\begin{array}{c}
a_{1}^{(1)}-a_{2}^{(1)} \\
\vdots \\
a_{1}^{(n)}-a_{2}^{(n)}
\end{array}\right)
$$

is nonsingular.

From conditions (i) and (ii), we have, for $j=1, \ldots, n$,

$$
\begin{aligned}
& \left\langle\alpha, a_{1}^{(j)}\right\rangle+w_{j}\left(a_{1}^{(j)}\right)=\left\langle\alpha, a_{2}^{(j)}\right\rangle+w_{j}\left(a_{2}^{(j)}\right), \text { and } \\
& \left\langle\alpha, a_{1}^{(j)}\right\rangle+w_{j}\left(a_{1}^{(j)}\right)<\langle\alpha, a\rangle+w_{j}(a), \text { for all } a \in S_{j}^{\prime} .
\end{aligned}
$$

Or,

$$
\begin{aligned}
& \left(a_{1}^{(j)}-a_{2}^{(j)}\right)^{T} \alpha=w_{j}\left(a_{2}^{(j)}\right)-w_{j}\left(a_{1}^{(j)}\right), \text { and } \\
& \left(a_{1}^{(j)}-a\right)^{T} \alpha<w_{j}(a)-w_{j}\left(a_{1}^{(j)}\right), \text { for all } a \in S_{j}^{\prime} .
\end{aligned}
$$

In simple notations, (25) can be written in the matrix form

$$
\bar{A} \alpha \leq d,
$$

where the rows of the matrix $\bar{A}$ consist of vectors of either $a_{1}^{(j)}-a_{2}^{(j)}$ or $a_{1}^{(j)}-a^{(j)}$ for certain $a \in S_{j}^{\prime} \backslash\left\{a_{1}^{(j)}, a_{2}^{(j)}\right\}$ and the corresponding entries of vector $d$ are either $w_{j}\left(a_{2}^{(j)}\right)-w_{j}\left(a_{1}^{(j)}\right)$ or $w_{j}\left(a^{(j)}\right)-w_{j}\left(a_{1}^{(j)}\right)$ respectively.

In view of (26), the search for $\alpha \in R^{n}$ in Step 1 can be organized as follows: Given any pair $\left(a_{1}^{(j)}, a_{2}^{(j)}\right)$ of two distinct points in each $S_{j}^{\prime}$, for $j=1, \ldots, n$, consider the combination of those pairs, 


$$
\left(a_{1}^{(1)}, a_{2}^{(1)}\right), \ldots,\left(a_{1}^{(n)}, a_{2}^{(n)}\right)
$$

when the matrix

$$
\left(\begin{array}{c}
a_{1}^{(1)}-a_{2}^{(1)} \\
\vdots \\
a_{1}^{(n)}-a_{2}^{(n)}
\end{array}\right)
$$

is nonsingular. The existence of $\alpha \in R^{n}$ for which the matrix inequality in (26) holds for this combination is known as the Phase I problem in Linear Programming for the feasibility of the constraints listed in (25). It is quite obvious that this problem is equivalent to the extreme value of the minimization problem,

$$
\begin{aligned}
& \min \epsilon, \\
& \bar{A} \alpha-\epsilon e \leq d, \quad e=(1, \ldots, 1)^{T}, \\
& \quad \epsilon \geq 0,
\end{aligned}
$$

in the variables $(\alpha, \epsilon)$ being 0 . The constraints of this minimization problem is always feasible, since for $\alpha=0$, we can always choose $\epsilon$ large enough to satisfy the matrix inequality in (28). Hence, the standard simplex algorithm in Linear Programming can be employed to solve this minimization problem. If the extreme value of $\epsilon$ fails to be zero, then for the combination in (27) in consideration there exists no $\alpha \in R^{n}$ together with which the matrix inequality (26) can be satisfied. When $\epsilon=0$ for certain $\alpha_{0} \in R^{n}$, then, obviously, $\alpha_{0}$ and the set of vectors in the combination meet our search requirement in Step 1. In this way, by examining all the possible combinations, we may obtain all the proper $\alpha$ 's along with their associated sets of vectors that we search for.

Apparently, this exhausting search procedure appears to be quite brutal and costly. However, in practice, this procedure can be made much more efficient with many special designs. For instance, to narrow down our search, we may test the feasibility conditions for all the possible pairs of distinct points in each individual $S_{j}^{\prime}$ in the first place, and only those pairs that survive the test can be considered as possible candidates for being an element in the combination in (27). To be more precise, for a fixed $j_{0}, 1 \leq j_{0} \leq n$, let $\left(a_{1}, a_{2}\right)$ be any pair of two distinct points in $S_{j_{0}}^{\prime}$. If this pair belongs to any successful combinations in (27) for which there exists $\alpha \in R^{n}$ such that both conditions (i) and (ii) are satisfied for all $j=1, . ., n$, then, by restricting conditions (i) and (ii) on $S_{j_{0}}^{\prime}$, this $\alpha$ must satisfy:

$$
\begin{aligned}
& (\mathrm{i})^{\prime}\left(a_{1}-a_{2}\right)^{T} \alpha=w^{\left(j_{0}\right)}\left(a_{2}\right)-w^{\left(j_{0}\right)}\left(a_{1}\right), \\
& (\mathrm{ii})^{\prime}\left(a_{1}-a\right)^{T} \alpha<w^{\left(j_{0}\right)}(a)-w^{\left(j_{0}\right)}\left(a_{1}\right) \text {, for all } a \in S_{j_{0}}^{\prime} .
\end{aligned}
$$


In other words, the constraints consisting of conditions $(i)^{\prime}$ and $(i i)^{\prime}$ must be feasible. We solve the Phase I problem of these constraints first, and the pair $\left(a_{1}, a_{2}\right)$ will be deleted for further considerations if those constraints fail to be feasible. In practice, this filtration can narrow down the search considerably.

The set $A_{\alpha}=\left\{\left(a_{1}^{(j)}, a_{2}^{(j)}\right), j=1, \ldots, n\right\}$ in (21) that satisfies (22) is known as a fine mixed cell of type $(1, \ldots ., 1)$ for the reason that each pair $\left(a_{1}^{(j)}, a_{2}^{(j)}\right)$ provides one independent direction $a_{1}^{(j)}-a_{2}^{(j)}$. The construction of those cells plays an important role when the polyhedral homotopy method is used to solve polynomial systems. The methods for the construction, including the one we described above, were discussed in $[11,12,38,39]$. At present, the most efficient technique for finding those cells remains undetermined. More rigorously, it appears that a plenty of room exists for major improvements of the existing methods.

We now discuss the method for solving the binomial system $(24)$ in $\left(C^{*}\right)^{n}$. Let

$$
v_{j}=a_{1}^{(j)}-a_{2}^{(j)}, \quad j=1, \ldots, n,
$$

and, with $y \in\left(C^{*}\right)^{n}$ in mind we rewrite the system (24) as

$$
\begin{array}{cc}
y^{v_{1}} & =b_{1}, \\
& \vdots \\
y^{v_{n}} & =b_{n},
\end{array}
$$

where $b_{j}=c_{j 2} / c_{j 1}$ for $j=1, \ldots, n$. Let

$$
V=\left[v_{1}^{T}\left|v_{2}^{T}\right| \cdots \mid v_{n}^{T}\right]
$$

and for brevity, write

$$
y^{V}=\left(y^{v_{1}}, \ldots, y^{v_{n}}\right) \quad \text { and } \quad b=\left(b_{1}, \ldots, b_{n}\right) .
$$

Then, (29) becomes,

$$
y^{V}=b .
$$

With this notation, it is easy to verify that for an $n \times n$ integer matrix $U$, the following holds,

$$
\left(y^{V}\right)^{U}=y^{(V U)} .
$$

Now, when the matrix $V$ in (30) is an upper triangular matrix, i.e.,

$$
V=\left[\begin{array}{cccc}
v_{11} & v_{12} & \cdots & v_{1 n} \\
0 & v_{22} & \cdots & v_{2 n} \\
\vdots & \ddots & \ddots & \vdots \\
0 & \cdots & 0 & v_{n n}
\end{array}\right]
$$


writing out the equation in (31) explicitly, we have,

$$
\begin{array}{ll}
y_{1}^{v_{11}} & =b_{1}, \\
y_{1}^{v_{12}} y_{2}^{v_{22}} & =b_{2}, \\
& \vdots \\
y_{1}^{v_{1 n}} y_{2}^{v_{2 n}} \cdots y_{n}^{v_{n n}} & =b_{n} .
\end{array}
$$

By forward substitutions, all the solutions of the system $(32)$ in $\left(C^{*}\right)^{n}$ can be found, and the total number of solutions is $\left|v_{11}\right| \times \cdots \times\left|v_{n n}\right|=|\operatorname{det} V|$.

In general, we may upper triangularize $V$ in (30) by the following process. Recall that the greatest common divisor $d$ of two nonzero integers $a$ and $b$, denoted by $\operatorname{gcd}(a, b)$, can be written as

$$
d=\operatorname{gcd}(a, b)=k a+l b
$$

for certain nonzero integers $k$ and $l$. Let

$$
M=\left[\begin{array}{cc}
k & l \\
-\frac{b}{d} & \frac{a}{d}
\end{array}\right] .
$$

We have $\operatorname{det}(M)=1$, and

$$
M\left[\begin{array}{l}
a \\
b
\end{array}\right]=\left[\begin{array}{cc}
k & l \\
-\frac{b}{d} & \frac{a}{d}
\end{array}\right]\left[\begin{array}{l}
a \\
b
\end{array}\right]=\left[\begin{array}{l}
d \\
0
\end{array}\right]
$$

Similar to using Givens rotation to produce zeros in a matrix for its $\mathrm{QR}$ factorization, the matrix $M$ may be used to upper triangularize $V$ as follows. For $v \in Z^{n}$, let $a$ and $b$ be its $i$ th and the $j$ th (nonzero) components where $i<j$, that is,

$$
v=\left[\begin{array}{c}
\vdots \\
a \\
\vdots \\
b \\
\vdots
\end{array}\right] \rightarrow j \text { th }
$$

With $d=\operatorname{gcd}(a, b)$, we let 
$i$ th $\quad j$ th

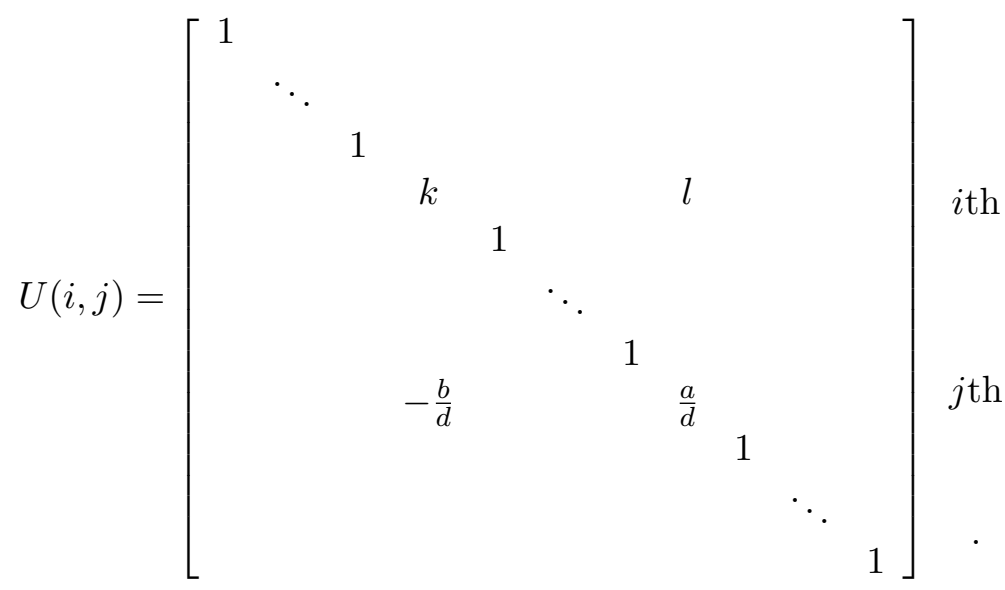

Evidently, $U(i, j)$ is an integer matrix with $|\operatorname{det}(U(i, j))|=1$ and

$$
U(i, j) v=\left[\begin{array}{c}
\vdots \\
d \\
\vdots \\
0 \\
\vdots
\end{array}\right] \quad \text { ith }
$$

Thus a series of matrices in the form of $U(i, j)$ in (33) may be used to successively produce zeros in the lower triangular part of the matrix $V$ in (30), resulting in an upper triangular matrix. In simple terms, we may construct an integer matrix $U$, as a product of those $U(i, j)$ 's, with $|\operatorname{det} U|=1$ and $U V$ is an upper triangular integer matrix.

Now, as mentioned above, the solutions of the system

$$
\left(z^{U}\right)^{V}=z^{U V}=b
$$

in $\left(C^{*}\right)^{n}$ can be found by forward substitutions, since $U V$ is an upper triangular integer matrix. And the total number of solutions in $\left(C^{*}\right)^{n}$ is

$$
|\operatorname{det}(U V)|=|\operatorname{det}(U)| \cdot|\operatorname{det}(V)|=|\operatorname{det}(V)| \text {. }
$$

By letting $y=z^{U}$ for each solution $z$ of $(34)$ in $\left(C^{*}\right)^{n}$, we obtain all the solutions of the system $(31)$ in $\left(C^{*}\right)^{n}$, and hence, solve the start system (24) in $\left(C^{*}\right)^{n}$ in Step 2. 


\section{Cheater's Homotopy}

The cheater's homotopy was originally designed in [25] (a similar procedure can be found in [32]) to deal with the problem when the polynomial was asked to be solved for several different values of the coefficients $c=\left(c_{1}, \ldots, c_{M}\right)$ (here $c$ may not include the coefficients of all the monomials). In other words, we think of $P(c, x)=0$ as a system with the same structure or sparsity.

Recall that a homotopy is considered to be successful when it possesses three properties: Property 0 (Triviality), Property 1 (Smoothness), and Property 2 (Accessibility). The idea of the cheater's homotopy is to establish Properties 1 and 2 theoretically by deforming a sufficiently generic system and then to cheat on Property 0 by using a preprocessing step. The amount of computation per preprocessing step may be large, but is shared among several solving characteristics of the problem.

We begin with an example. Let $P\left(x_{1}, x_{2}\right)=\left(p_{1}\left(x_{1}, x_{2}\right), p_{2}\left(x_{1}, x_{2}\right)\right)$ be the system

$$
\begin{aligned}
& p_{1}\left(x_{1}, x_{2}\right)=x_{1}^{3} x_{2}^{2}+c_{1} x_{1}^{3} x_{2}+x_{2}^{2}+c_{2} x_{1}+c_{3}=0, \\
& p_{2}\left(x_{1}, x_{2}\right)=c_{4} x_{1}^{4} x_{2}^{2}-x_{1}^{2} x_{2}+x_{2}+c_{5}=0 .
\end{aligned}
$$

This is a system of two polynomial equations in two unknowns $x_{1}$ and $x_{2}$. We want to solve this system of equations several times, for various specific choices of $c=\left(c_{1}, \ldots, c_{5}\right)$. It turns out that for any choice of coefficients $c$, system (35) has no more than 10 isolated solutions in $C^{2}$. (This may be considered as a consequence of applying Theorem 2.3 in Section 2, since the mixed volume of the system is 10.) The total degree of the system is $6 \times 5=30$, meaning that the classical homotopy (2) using the start system $Q(x)=0$ in (3) will produce $d=30$ paths, beginning at 30 trivial starting points. Thus there are (at least) 20 extraneous paths.

The cheater's homotopy continuation approach begins by solving (35) with randomly chosen complex coefficients $\bar{c}=\left(\bar{c}_{1}, \ldots, \bar{c}_{5}\right)$; let $X^{*}$ be the set of 10 solutions. Subsequently, for each choice of coefficients $c=\left(c_{1}, \ldots, c_{5}\right)$ for which the system (35) needs to be solved, we use the homotopy continuation method to follow a straight-line homotopy, as in (2), from the system with coefficient $\bar{c}$ to the system with coefficient $c$, and we follow the 10 paths beginning at the 10 elements of $X^{*}$. Thus Property 0, the existence of trivial starting points, is satisfied. The fact that Properties 1 and 2 are also satisfied is the content of Theorem 5.1 below. Thus for each fixed $c$, all 10 (or fewer) isolated solutions of (35) lie at the end of 10 smooth homotopy paths beginning at the seeds in $X^{*}$. After the initial step of finding the seeds, the complexity of all further solving of (35) is proportional to the number of solutions 10, rather than the total degree 30 . 
A statement of the theoretical result we need follows. Let

$$
R(c, x)=\left\{\begin{array}{c}
r_{1}\left(c_{1}, \ldots, c_{M}, x_{1}, \ldots, x_{n}\right)=0 \\
\vdots \\
r_{n}\left(c_{1}, \ldots, c_{M}, x_{1}, \ldots, x_{n}\right)=0
\end{array}\right.
$$

be a system of polynomial equations in the variables $c=\left(c_{1}, \ldots, c_{M}\right)$ and $x=\left(x_{1}, \ldots, x_{n}\right)$. For each choice of $c=\left(c_{1}, \ldots, c_{M}\right)$ in $C^{M}$, this is a system of polynomial equations in the variables $x_{1}, \ldots, x_{n}$.

Theorem 5.1. Let $c$ belong to $C^{M}$. There exists an open dense fullmeasure subset $U$ of $C^{n+M}$ such that for $(\bar{b}, \bar{c})=\left(b_{1}^{*}, \ldots, b_{n}^{*}, c_{1}^{*}, \ldots, c_{M}^{*}\right) \in U$, the following holds:

(a) The set $X^{*}$ of solutions $x=\left(x_{1}, \ldots, x_{n}\right)$ of the system

$$
G(\bar{b}, \bar{c}, x)=\left\{\begin{array}{c}
g_{1}\left(x_{1}, \ldots, x_{n}\right)=r_{1}\left(c_{1}^{*}, \ldots, c_{M}^{*}, x_{1}, \ldots, x_{n}\right)+b_{1}^{*}=0 \\
\vdots \\
g_{n}\left(x_{1}, \ldots, x_{n}\right)=r_{n}\left(c_{1}^{*}, \ldots, c_{M}^{*}, x_{1}, \ldots, x_{n}\right)+b_{n}^{*}=0
\end{array}\right.
$$

consists of isolated nonsingular solutions.

(b) Properties 1 and 2 (Smoothness and Accessibility) hold for the homotopy

$$
H(x, t)=(1-t) G(\bar{b}, \bar{c}, x)+t R(c, x)=0
$$

for any $c \in C^{M}$. It follows that for any fixed $c$, every solution of $R(c, x)=0$ is reached by a path of $H(x, t)=0$ beginning at a point of $X^{*}$.

A proof of this theorem can be found in [25]. As a special case hiding in the proof, when all the components $r_{j}(c, x)$ of $R(c, x)$ have constant terms and their coefficients are members of the coordinates of $c=\left(c_{1}, \ldots, c_{M}\right)$, as in the system $(35)$, then $\bar{b}=\left(b_{1}^{*}, \ldots, b_{n}^{*}\right)$ in the theorem can be replaced by $\bar{b}=(0, \ldots, 0)$. This result will be used later.

The theorem is used as part of the following procedure. Let $R(c, x)=0$, as in (36), denote the system to be solved for various values of the coefficients $c$.

\section{Cheater's Homotopy Procedure.}

Step 1. Choose complex numbers $(\bar{b}, \bar{c})=\left(b_{1}^{*}, \ldots, b_{n}^{*}, c_{1}^{*}, \ldots, c_{M}^{*}\right)$ at random and solve $G(\bar{b}, \bar{c}, x)=0$ in (37). Let $X^{*}$ denote the set of $d_{0}$ solutions we found. 
Step 2. For each new choice of coefficients $c=\left(c_{1}, \ldots, c_{M}\right)$, follow the $d_{0}$ paths defined by the zero set of the homotopy $H(x, t)=0$ in $(38)$, beginning at the points in $X^{*}$, to find all solutions of $R(c, x)=0$.

In Step 1 above, solve $G(\bar{b}, \bar{c}, x)=0$ in (37) may sometimes be computationally expensive for randomly chosen $c=\left(c_{1}^{*}, \ldots, c_{M}^{*}\right)$. It is desirable that, at least in certain special occasions, those coefficients are allowed to be specific so that the solutions of the equation $G(\bar{b}, \bar{c}, x)=0$ is obvious. To illustrate, consider the linear system

$$
\begin{gathered}
c_{11} x_{1}+\cdots+c_{1 n} x_{n}=b_{1}, \\
\vdots \\
c_{n 1} x_{1}+\cdots+c_{n n} x_{n}=b_{n},
\end{gathered}
$$

as a system consisting of polynomial equations with degree one each. For randomly chosen $c_{i j}$ 's, the system in (39) has a unique solution which is not available right away. However, if we are allowed to let $c_{i j}=\delta_{i j}$ (the Kronecker delta), the solution is obvious.

For this purpose, an alternative is suggested in [27]. When a system $R(c, x)=0$ with a specific $c^{0}$, not necessarily randomly chosen, is solved, then for any parameter $c \in C^{M}$ consider the nonlinear homotopy

$$
H(a, x, t)=R\left((1-[t-t(1-t) a]) c^{0}+(t-t(1-t) a) c, x\right)=0 .
$$

It was shown in [27] that this homotopy satisfies all three Properties 0, 1, 2 (Triviality, Smoothness, Accessibility, respectively) for generically chosen $a \in C$ under the natural assumption that for generic $c, R(c, x)=0$ has the same number of isolated zeros in $C^{n}$ and this number agrees with that of $R\left(c^{0}, x\right)=0$. This idea was used in [26] for solving the indirect position problem for revolt-joint kinematic manipulators more efficiently.

While the purposes are different, the machinery of the cheater's homotopy method described above fits our procedure for solving a polynomial system $P(x)=\left(p_{1}(x), \ldots, p_{n}(x)\right) \in C[x]$ with fixed coefficients by the polyhedral homotopies quite well. As described in Section 3, we first augment the monomial $x^{0}(=1)$ to those $p_{i}$ 's which do not have constant terms. Followed by choosing coefficients of all the monomials at random, a new system $Q(x)=0$ is obtained. We then solve all the isolated solutions of this new system $Q(x)=0$ by establishing the polyhedral homotopy $\hat{Q}(x, t)=0$ in (10).

Now, after $Q(x)=0$ is solved, consider the linear homotopy

$$
H(x, t)=(1-t) Q(x)+t P(x)=0 .
$$

By Theorem 5.1, Properties 1 and 2 (Smoothness and Accessibility) hold for this homotopy. Notice that since every component $q_{j}(x)$ of $Q(x)$, for $j=$ 
$1, \ldots, n$ has constant term, $\bar{b}=\left(b_{1}^{*}, \ldots, b_{n}^{*}\right)$ in the theorem can be replaced by $\bar{b}=(0, \ldots, 0)$ as we mentioned before. Moreover, because of the availability of all the isolated solutions of $Q(x)=0$, Property 0 also holds. Therefore, every isolated zeros of $P(x)$ lies at the end of a homotopy path of $H(x, t)=0$ in (40), starting from an isolated solutions of $Q(x)=0$.

\section{Further Considerations}

For the purpose of discussing further developments of the polyhedral homotopy method described in Sections 2-5 for finding all isolated zeros of a given polynomial system $P(x)=\left(p_{1}(x), \ldots, p_{n}(x)\right)$ in $C^{n}$ with $\operatorname{supp}\left(p_{j}\right)=S_{j}$, for $j=1, \ldots, n$, we outline the major steps in brief terms as follows:

(0) Choose a polynomial system $Q(x)=\left(q_{1}(x), \ldots, q_{n}(x)\right)$ with $\operatorname{supp}\left(q_{j}\right)=$ $S_{j} \cup\{0\}, j=1, \ldots, n$, and with randomly chosen coefficients. Let $k=$ $\mathcal{M}\left(\mathcal{S}_{\infty} \cup\{\prime\}, \ldots, \mathcal{S} \backslash \cup\{\prime\}\right)$, the mixed volume of the support $\left(S_{1} \cup\{0\}, \ldots, S_{n} \cup\right.$ $\{0\})$.

(A) Set up the polyhedral homotopy $\hat{Q}(x, t):\left(C^{*}\right)^{n} \times[0,1] \rightarrow\left(C^{*}\right)^{n}$ for $Q(x)$ with $\hat{Q}(x, 1)=Q(x)$. Namely, for $j=1, \ldots, n$,

$$
q_{j}(x)=\sum_{a} \bar{c}_{j, a} x^{a} \quad \Longrightarrow \quad \hat{q}_{j}(x)=\sum_{a} \bar{c}_{j, a} x^{a} t^{w_{j}(a)} .
$$

(B) Find the finite subset $B$ in $R^{n}$ given in Proposition 3.2, and for each $\alpha \in B$, define the homotopy

$$
H_{\alpha}(y, t) \equiv t^{-\beta} \hat{Q}\left(y t^{\alpha}, t\right)=0,
$$

where $\beta=\left(\beta_{1}, \ldots, \beta_{n}\right)$ and $\beta_{j}$ is the lowest order in $t$ among all the terms in $\hat{q}_{j}\left(y t^{\alpha}, t\right)$.

(C) Solve the start system $H_{\alpha}(y, 0)=0$ in $\left(C^{*}\right)^{n}$, and follow homotopy paths of $H_{\alpha}(y, t)=0$ with starting points in $\left(C^{*}\right)^{n}$ for all $\alpha \in B, k$ paths of them in total, to find all the isolated zeros of $Q(x)$.

(D) Use the linear homotopy

$$
H(x, t)=(1-t) Q(x)+t P(x)=0
$$

to find all isolated zeros of $P(x)$ in $C^{n}$ by following $k$ homotopy paths starting from isolated zeros of $Q(x)$.

Now here are some remarks as regards to further developments of the method: 
(i) As we can see, in order to find all the isolated zeros of $P(x)$ in $C^{n}$ there are $k$ homotopy paths in both Steps (C) and (D), and hence $2 k$ in total, need to be followed. This work may be reduced by half by the following strategy as suggested in [17]: First of all, at Step (A), we let

$$
\tilde{Q}(x, t)=(1-t) Q(x)+t P(x)
$$

and set up the polyhedral homotopy $\bar{Q}(x, t)=\left(\bar{q}_{1}(x, t), \ldots, \bar{q}_{n}(x, t)\right)=0$ for $\tilde{Q}(x, t)$ instead with the same variable $t$. More precisely, for generic $w_{j}: S_{j} \cup\{0\} \rightarrow R, j=1, \ldots, n$,

$$
\left.\begin{array}{l}
p_{j}(x)=\sum_{a} c_{j, a}^{*} x^{a} \\
q_{j}(x)=\sum_{a} \bar{c}_{j, a} x^{a}
\end{array}\right\} \Longrightarrow \bar{q}_{j}(x, t)=\sum_{a}\left[(1-t) \bar{c}_{j, a}+t c_{j, a}^{*}\right] x^{a} t^{w_{j}(a)} .
$$

Apparently, we have $\bar{Q}(x, 1)=P(x)$ and for each $t$ in $[0,1], \bar{Q}(x, t)$ and $\tilde{Q}(x, t)$ have the same support. Moreover, since

$$
\tilde{Q}(x, t)=Q(x)+t(P(x)-Q(x)),
$$

it is clear that, for any $\alpha \in R^{n}$, the lowest order terms in $t$ of both $\hat{q}_{j}\left(y t^{\alpha}, t\right)$ and $\bar{q}_{j}\left(y t^{\alpha}, t\right)$ are the same. Hence the finite subset $B$ in Step (B) stays invariant, and the start system of the homotopy

$$
\bar{H}_{\alpha}(y, t) \equiv t^{-\beta} \bar{Q}\left(y t^{\alpha}, t\right)=0
$$

is the same as that of $H_{\alpha}(y, t)=0$ in (41). Here, again, $\beta=\left(\beta_{1}, \ldots, \beta_{n}\right)$ and $\beta_{j}$ is the lowest order in $t$ among all the terms in $\bar{q}_{j}\left(y t^{\alpha}, t\right)$. Thus, when nonsingular solutions of $H_{\alpha}(y, 0)=0$ in $\left(C^{*}\right)^{n}$ are available in Step (C), we may follow those homotopy paths of $\bar{H}_{\alpha}(y, t)=0$ in (43) instead with those starting points. Those paths, $k$ of them in total for all $\alpha \in B$, will lead to all the isolated zeros of $P(x)$ at $t=1$ without employing the linear homotopy in Step (D). In this way we only need to follow $k$ homotopy paths in total.

A major disadvantage of this approach is the possibility of losing a degree of stability in the curve following scheme for those new paths. We may save the work of following the extra number of paths at the expense of reducing the speed of following the remaining number of paths. At present, the magnitude of the cost of this trade-off remains undetermined. For clearance, an intensive investigation of this approach on a vast variety of polynomial systems is required. 
(ii) The mixed volume $k=\mathcal{M}\left(S_{1} \cup\{0\}, \ldots, S_{n} \cup\{0\}\right)$, which represents the number of paths we need to follow, provides an upper bound for the root count of the system $P(x)$ in $C^{n}$. This bound may not be exact, meaning that we might follow extraneous paths as the following example shows: Consider the system

$$
P\left(x_{1}, x_{2}\right)=\left\{\begin{array}{l}
p_{1}\left(x_{1}, x_{2}\right)=a x_{2}+b x_{2}^{2}+c x_{1} x_{3} \\
p_{2}\left(x_{1}, x_{2}\right)=d x_{1}+e x_{1}^{2}+f x_{1}^{3} x_{2} .
\end{array}\right.
$$

For generic coefficients $\{a, b, c, d, e, f\}$, this system has six isolated zeros in $C^{2}$ and three isolated zeros in $\left(C^{*}\right)^{2}$. However, its augmented system

$$
Q\left(x_{1}, x_{2}\right)=\left\{\begin{array}{l}
q_{1}\left(x_{1}, x_{2}\right)=\epsilon_{1}+a x_{2}+b x_{2}^{2}+c x_{1} x_{3} \\
q_{2}\left(x_{1}, x_{2}\right)=\epsilon_{2}+d x_{1}+e x_{1}^{2}+f x_{1}^{3} x_{2}
\end{array}\right.
$$

has eight isolated zeros in $\left(C^{*}\right)^{2}$, since the mixed volume of $Q\left(x_{1}, x_{2}\right)$ is eight. So eight paths in either the homotopy $H(x, t)=0$ in (42) or the homotopy $\bar{H}_{\alpha}(y, t)=0$ in (43) need to be traced to find all six isolated zeros in $C^{2}$ of $P\left(x_{1}, x_{2}\right)$ in (44), and two of them are obviously extraneous.

In [18], Huber and Sturmfels gave a tighter upper bound for the root count of the polynomial system $P(x)$ in $C^{n}$ by introducing the stable mixed cells. The sum of the mixed volumes of those stable mixed cells is called the stable mixed volume, and the root count of $P(x)$ in $C^{n}$ is bounded above by this number. By the way the stable mixed volume, denoted by $\mathcal{S M}\left(S_{1}, \ldots, S_{n}\right)$, is defined, it satisfies

$$
\mathcal{M}\left(S_{1}, \ldots, S_{n}\right) \leq \mathcal{S} \mathcal{M}\left(S_{1}, \ldots, S_{n}\right) \leq \mathcal{M}\left(S_{1} \cup\{0\}, \ldots, S_{n} \cup\{0\}\right) .
$$

Based on this tighter upper bound, a homotopy algorithm was suggested in [18], so that fewer homotopy paths need to be followed.

A drawback of this method is the drastic increase of the computation effort in determining the stable mixed cells. This drawback is overcome in [19].

(iii) In some occasions, the polyhedral homotopy method is capable of taking advantages of the special structures characterized by certain polynomial systems. In [17], a more general version of the algorithm was presented when some of the $p_{j}$ 's share the same supports and the algorithm can be made much more efficient by taking this special structure into consideration. In [37], symmetric polyhedral homotopies were constructed to deal with certain symmetric polynomial systems. 
(iv) Several software packages dedicated to solving polynomial systems by homotopy continuation methods are publicly available. HOMPACK [33] and CONSOL [30] are written in FORTRAN 77. HOMPACK is a general package for homotopy continuation with a polynomial driver. It has been parallelized to various architectures $[3,14]$. The code for CONSOL is contained in [30], Appendix 6.

The polyhedral homotopy method was first implemented in PHC [38] and Pelican [15]. The package PHC written in Ada, along with its FORTRAN version developed by the author and his students, offers a wide variety of root-counting and homotopy methods. The performance of these codes on a large collection of more than eighty polynomial systems coming from a wide variety of application fields [9] provides practical evidence that the newly developed methods constitute a powerful general purpose solver. Nonetheless, a more efficient and user-friendly black-box solver for even more extended applications is still under development. It is hoped that the polyhedral homotopy method would become even more powerful as well as more applicable with a better understanding of convex geometry and a clever use of linear programming techniques.

The modern scientific computing is marked by the advent of vector and parallel computers and the search for algorithms that are to a large extent naturally parallel. A great advantage of the homotopy continuation algorithm for solving polynomial system is that it is to a large degree parallel, in the sense that each isolated zero can be computed independently. This natural parallelism makes the method an excellent candidate for a variety of architectures. In this respect, it stands in contrast to the highly serial Gröbner bases method. We wish to return to this subject in the foreseeing future.

\section{REFERENCES}

1. E. L. Allgower and K. Georg, Numerical Continuation Methods, an Introduction, Springer Series in Comput. Math., Vol. 13, Springer-Verlag, Berlin Herdelberg, New York, 1990.

2. E. L. Allgower and K. Georg, Continuation and path following, in: Acta Numerica. 1993, A. Iserles, ed., Cambridge Univ. Press, Cambridge, 1993, pp.1-64.

3. D. C. S. Allison, A. Chakraborty and L. T. Watson, Granularity issues for solving polynomial systems via globally convergent algorithms on a hypercube, J. Supercomputing 3 (1989), 5-20.

4. D. N. Bernshteín, The number of roots of a system of equations, Funct. Anal. Appl. 9 (1975), 183-185. 
5. B. Buchberger, Gröbner basis: An algorithmic method in polynomial ideal theory, in: Multidimensional System Theory, N.K. Bose, ed., D. Reidel Publishing Company, Dordrecht, Boston, Lancaster, 1985, pp.184-232.

6. J. Canny and J. M. Rojas, An optimal condition for determining the exact number of roots of a polynomial system, in: Proceedings of the 1991 International Symposium on Symbolic and Algebraic Computation, ACM, 1991, pp.96-101.

7. S. N. Chow, J. Mallet-Paret and J. A. Yorke, Finding zeros of maps: homotopy methods that are constructive with probability one, Math. Comp. 32 (1978), 887-899.

8. S. N. Chow, J. Mallet-Paret and J. A. Yorke, Homotopy method for locating all zeros of a system of polynomials, in: Functional Differential Equations and Approximation of Fixed Points, H. O. Peitgen and H. O. Walther, eds., Lecture Notes in Mathematics, Vol. 730, Springer-Verlag, Berlin, Heidelberg, 1979, pp.77-88.

9. H. H. Chung, Polyhedral Homotopy and its Applications to Polynomial System Solving, Ph.D. dissertation, Michigan State University, 1998.

10. F. J. Drexler, Eine Methode zur Berechnung sämtlicher Lösungen von Polynomgleichungssystemen, Numer. Math. 29 (1977), 45-58.

11. I. Emiris, Sparse Elimination and Applications in Kinematics, Ph.D. thesis, Computer Science Division, Dept. of Electrical Engineering and Computer Science, University of California (Berkeley), 1994.

12. I. Emiris and J. Canny, Efficient incremental algorithms for the sparse resultant and the mixed volume, J. Symbolic Comput. 20 (1995), 117-149.

13. C. B. Garcia and W. I. Zangwill, Finding all solutions to polynomial systems and other systems of equations, Math. Programming 16 (1979), 159-176.

14. S. Harimoto and L. T. Watson, The granularity of homotopy algorithms for polynomial systems of equations, in: Parallel Processing for Scientific Computing, G. Rodrigue, ed., SIAM, 1989, pp.115-120.

15. B. Huber, Pelican manual, available via the author's Web page.

16. B. Huber, Solving Sparse Polynomial Systems, Ph.D. dissertation, Cornell University, 1996.

17. B. Huber and B. Sturmfels, A polyhedral method for solving sparse polynomial systems, Math. Comp. 64 (1995), 1541-1555.

18. B. Huber and B. Sturmfels, Bernshtein's theorem in affine space, Discrete Comput. Geom. 7 (1997), 137-141.

19. T. Gao, T.Y. Li and X. Wang, Finding isolated zeros of polynomial systems in $C^{n}$ with stable mixed volumes, to appear in J. Symbolic Comput..

20. A. G. Khovanskií, Newton polyhedra and the genus of complete intersections, Funct. Anal. Appl. 12 (1978), 38-46. 
21. A. G. Kushnirenko, Newton polytopes and the Bèzout theorem, Funct. Anal. Appl. 10 (1976), 233-235.

22. T. Y. Li, On Chow, Mallet-Paret and Yorke homotopy for solving systems of polynomials, Bull. Inst. Math. Acad. Sinica 11 (1983), 433-437.

23. T. Y. Li, Numerical solution of multivariate polynomial systems by homotopy continuation methods, Acta Numerica 6 (1997), 399-436.

24. T. Y. Li and T. Sauer, A simple homotopy for solving deficient polynomial systems, Japan J. Indust. Appl. Math. 6 (1989), 409-419.

25. T. Y. Li, T. Sauer and J. A. Yorke, The cheater's homotopy: an efficient procedure for solving systems of polynomial equations, SIAM J. Numer. Anal. 26 (1989), 1241-1251.

26. T. Y. Li and X. Wang, A homotopy for solving the kinematics of the most general six-and-five-degree of freedom manipulators, Proc. of ASME Conference on Mechanisms, D1-Vol. 25 (1990), 249-252.

27. T. Y. Li and X. Wang, Nonlinear homotopies for solving deficient polynomial systems with parameters, SIAM J. Numer. Anal. 29 (1992), 1104-1118.

28. T. Y. Li and X. Wang, The BKK root count in $C^{n}$, Math. Comp. 65 (1996), 1477-1484.

29. A. P. Morgan, A homotopy for solving polynomial systems, Appl. Math. Comput. 18 (1986), 173-177.

30. A. P. Morgan, Solving Polynomial Systems Using Continuation for Engineering and Scientific Problems, Prentice-Hall, Englewood Cliffs, N. J., 1987.

31. A. P. Morgan and A. J. Sommese, A homotopy for solving general polynomial systems that respect $m$-homogeneous structures, Appl. Math. Comput. 24 (1987), 101-113.

32. A. P. Morgan and A. J. Sommese, Coefficient-parameter polynomial continuation, Appl. Math. Comput. 29 (1989), 123-160. Errata: Appl. Math. Comput. 51 (1992), 207.

33. A. P. Morgan, A. J. Sommese and L. T. Watson, Finding all isolated solutions to polynomial systems using HOMPACK, ACM Trans. Math. Software 15 (1989), 93-122.

34. J. M. Rojas, A convex geometric approach to counting the roots of a polynomial system, Theoret. Comput. Sci. 133 (1994), 105-140.

35. J. M. Rojas and X. Wang, Counting affine roots of polynomial systems via pointed Newton polytopes, J. Complexity 12 (1996), 116-133.

36. I. R. Shafarevich, Basic Algebraic Geometry, Springer-Verlag, New York, 1977.

37. J. Verschelde and K. Gatermann, Symmetric Newton polytopes for solving sparse polynomial systems, Adv. in Appl. Math. 16 (1995), 95-127. 
38. J. Verschelde, PHC and MVC: two programs for solving polynomial systems by homotopy continuation, 1995. Presented at the PoSSo Workshop on Software, Paris. Available by anonymous ftp to ftp.cs.kuleuven.ac.be in the directory /pub/NumAnal-ApplMath/PHC.

39. J. Verschelde, K. Gatermann and R. Cools, Mixed-volume computation by dynamic lifting applied to polynomial system solving, Discrete Comput. Geom. 16 (1996), 69-112.

40. A. H. Wright, Finding all solutions to a system of polynomial equations, Math. Comp. 44 (1985), 125-133.

41. W. Zulener, A simple homotopy method for determining all isolated solutions to polynomial systems, Math. Comp. 50 (1988), 167-177.

Department of Mathematics, Michigan State University

East Lansing, MI 48824, U.S.A.

E-mail: li@math.msu.edu 